INTERNATIONAL JOURNAL OF ROBUST AND NONLINEAR CONTROL

Int. J. Robust Nonlinear Control 2009; 19:1770-1786

Published online 5 November 2008 in Wiley InterScience (www.interscience.wiley.com). DOI: 10.1002/rnc.1389

\title{
Kalman filtering over unreliable communication networks with bounded Markovian packet dropouts
}

\author{
Nan $\mathrm{Xiao}^{1}$, Lihua $\mathrm{Xie}^{1, *, \dagger}$ and Minyue $\mathrm{Fu}^{2}$ \\ ${ }^{1}$ School of Electrical and Electronic Engineering, Nanyang Technological University, Singapore 639798, Singapore \\ ${ }^{2}$ School of Electrical Engineering and Computer Science, University of Newcastle, \\ Callaghan, NSW 2308, Australia
}

\begin{abstract}
SUMMARY
We address the peak covariance stability of time-varying Kalman filter with possible packet losses in transmitting measurement outputs to the filter via a packet-based network. The packet losses are assumed to be bounded and driven by a finite-state Markov process. It is shown that if the observability index of the discrete-time linear time-invariant (LTI) system under investigation is one, the Kalman filter is peak covariance stable under no additional condition. For discrete LTI systems with observability index greater than one, a sufficient condition for peak covariance stability is obtained in terms of the system dynamics and the probability transition matrix of the Markov chain. Finally, the validity of these results is demonstrated by numerical simulations. Copyright (C) 2008 John Wiley \& Sons, Ltd.
\end{abstract}

Received 30 January 2008; Revised 14 June 2008; Accepted 9 September 2008

KEY WORDS: Kalman filtering; packet losses; peak covariance stability; sojourn times

\section{INTRODUCTION}

Kalman filtering [1,2] plays an essential role in systems theory and has found a wide range of applications in engineering and non-engineering systems. A number of properties including stability and convergence of Kalman filtering have been well understood. For linear time-invariant (LTI) systems, it is well known that the Kalman filter converges to the steady-state filter under very mild hypotheses on some detectable and stabilizable conditions of the original system. Recently,

\footnotetext{
*Correspondence to: Lihua Xie, School of Electrical and Electronic Engineering, BLK S2, Nanyang Technological University, Nanyang Avenue, Singapore 639798, Singapore.

${ }^{\dagger}$ E-mail: elhxie@ntu.edu.sg

Contract/grant sponsor: Agency for Science, Technology and Research of Singapore; contract/grant number: 0521010037

Contract/grant sponsor: National Science Foundation of China; contract/grant number: 60828006

Contract/grant sponsor: National Science Foundation of China-Guangdong Joint Foundation; contract/grant number: U0735003
}

Copyright (C) 2008 John Wiley \& Sons, Ltd. 
there has been a lot of interest on networked systems and sensor networks due to increasing applications of networks in engineering systems. In sensor networks, Kalman filters are frequently used in accomplishing estimation task [3,4] and have given birth to several methods in solving the sensor coverage and sensor selection problems $[5,6]$. However, the recent trend of utilizing networks for transmitting measurement data also introduces some interesting new problems due to the unreliable characteristics of networks such as random transmission delays and possible packet losses [7]. These network uncertainties, unlike the uncertainties in dynamical systems studied in the past two decades, e.g. [8-10], are random in nature and have attracted considerable attention.

When the Kalman filter is implemented over network with packet dropouts, the evolution of filtering error covariance for an unstable dynamical system generally depends on not only system structure properties such as stabilizability and detectability, similar to the classical Kalman filter case, but also packet-loss characteristics. Therefore, the filtering problem for networked systems has attracted a great deal of attention recently. Kalman filtering for lossy sensor data was considered in [11] based on a Markovian jump linear system modeling approach and three different models were presented there. Sinopoli et al. [12] addressed Kalman filtering with i.i.d. Bernoulli losses and showed that a critical value for the arrival rate exists such that the mean filtering error covariance is bounded for any arrival rate greater than that critical value. The stability analysis of Kalman filtering with binary Markovian packet losses can be found in $[13,14]$ where the notion of peak covariance stability was first introduced in [15]. It is noted in $[13,15]$ that the given sufficient condition for the peak covariance stability involves an estimation of an upper bound of the solution of standard Riccati recursion.

However, in the aforementioned studies on filtering the number of consecutive packet losses can be infinite, which may not be practical and can lead to conservative results. For networked systems, control problems with finite-length packet losses have been investigated in [16,17]. Generally, there are two motivations for bounded packet-loss modeling. First of all, in fault-free networked systems, infinite-length consecutive packet dropouts are usually unrealistic. On the other hand, fault detection and fault tolerant systems can be implemented for real networked systems [18], which can effectively avoid the performance degradation and instability of the whole system due to excessive consecutive packet losses.

To the best of our knowledge, the stability issue related to the Kalman filtering for networked systems with bounded Markovian dropouts remains open. We note that the stability studied in [13-15] is in fact in terms of mean filtering error covariance, and thus the term-peak covariance stability in the mean sense would be more precise. We still use peak covariance stability here for the consistency of the present paper with the aforementioned ones. In the sequel, the sojourn times of loss cycle (a period of successive packet dropouts) and normal cycle (a period of successive packet receipts) are defined similarly to the binary dropout case $[12,13]$. The main results of the present paper include: (1) a less conservative method for estimating the upper bound of the solution of the standard Riccati recursion and (2) a sufficient condition for the peak covariance stability that is related to the observability index and the dynamics of the underlying system as well as the probability transition matrix of the Markov chain that describes the packet-loss process.

The remainder of this paper is organized as follows. The time-varying Kalman filtering problem with bounded Markovian dropouts is formulated in Section 2. Then basic lemmas as well as conditions for peak covariance stability are presented in Section 3. Section 4 includes two numerical examples, followed by some conclusions in Section 5.

Notation: $:=$ means 'defined as'. The superscript ' denotes the transpose of vector or matrix. $M^{-1}, \rho(M)$ represent, respectively, the inverse and the spectral radius of square matrix $M . \mathscr{R}^{n}, \mathscr{N}$, 
and $\mathscr{N}_{0}$ stand for the $n$-dimensional Euclidean space and the sets of positive and non-negative integers, respectively. When $X$ and $Y$ are real symmetric matrices, the notation $X \geqslant Y(X>Y)$ indicates that $X-Y$ is positive semidefinite(positive definite), and $\mathscr{S}^{n}$ denotes the set of $n \times n$ positive semidefinite real matrices. $I_{n}$ refers to the $n \times n$ identity matrix, and $I$ and 0 represent respectively identity matrix and zero matrix with compatible dimensions. $\delta_{i j}$ is the Kronecker delta function; $\operatorname{col}(x, y)$ denotes a column vector with entries $x, y .\|\cdot\|$ represents the Euclidean norm for vectors or the corresponding induced norm for matrices. Furthermore, let $(\Omega, \Xi, \operatorname{Pr})$ be a complete probability space and $E(\cdot)$ stands for the mathematical expectation operator. The inner product is defined by $\langle x, y\rangle:=E\left(x y^{\prime}\right)$ for column random vectors $x, y$.

\section{PROBLEM FORMULATION}

Consider a standard discrete-time LTI system:

$$
\begin{aligned}
x_{t+1} & =A x_{t}+B w_{t}, \quad t \geqslant 0 \\
y_{t} & =C x_{t}+v_{t}
\end{aligned}
$$

where $x_{t} \in \mathscr{R}^{n}$ and $y_{t} \in \mathscr{R}^{m}$ are the state and output of the system. The process noise $w_{t} \in \mathscr{R}^{p}$ and the measurement noise $v_{t} \in \mathscr{R}^{m}$ are vector-valued zero-mean white-noise processes, while the initial state $x_{0}$ is a random variable with zero mean and covariance matrix $\Sigma_{0} \geqslant 0$, and is independent of the sequences $\left\{w_{t}\right\},\left\{v_{t}\right\}$. The above assumptions can be compactly expressed as

$$
\left\langle\left[\begin{array}{c}
w_{i} \\
v_{i} \\
x_{0}
\end{array}\right],\left[\begin{array}{c}
w_{j} \\
v_{j} \\
x_{0} \\
1
\end{array}\right]\right\rangle=\left[\begin{array}{cccc}
Q \delta_{i j} & S \delta_{i j} & 0 & 0 \\
S^{\prime} \delta_{i j} & R \delta_{i j} & 0 & 0 \\
0 & 0 & \Sigma_{0} & 0
\end{array}\right], \quad Q>0, \quad R>0
$$

We also assume that $\left\{w_{t}\right\}$ and $\left\{v_{t}\right\}$ are uncorrelated, i.e. $S=0$ in (3). Moreover, $A, B, C, Q$, and $R$ are constant and known matrices with compatible dimensions. In this paper, $(A, B)$ is stabilizable and $(A, C)$ is observable with observability index $I_{0}$. Here $I_{\mathrm{O}}$ is defined to be the smallest integer such that $\operatorname{rank}\left[C^{\prime}, A^{\prime} C^{\prime}, \ldots,\left(A^{\prime}\right)^{I_{0}-1} C^{\prime}\right]=n$.

On account of reduced wiring and ease of system maintenance/diagnosis, there have been significant interests in transmitting sensor/controller data through networks, especially wireless networks. While networked systems have clear advantages, they also introduce new problems such as packet losses in transmission. Suppose there exists a communication channel between sampler and filter, and a single packet containing the data of system output is unreliably transmitted at each time step without quantization error. Let $\left\{i_{k}, k \geqslant 0\right\}$ be a strictly monotonically increasing subset of $\mathscr{N}_{0}$, representing the sequence of time instants at which the data packets are successfully transmitted from the sampler at the system to the filter. Without loss of generality, set $i_{0}=0$. The packet-loss process is defined as

$$
\eta\left(i_{k}\right):=i_{k+1}-i_{k}-1, \quad k \geqslant 0
$$

In the same way as in [17], we assume that the packet-loss process is driven by a timehomogeneous Markov chain on a complete probability space $(\Omega, \Xi, \operatorname{Pr})$ with finite-state range set 
$\mathscr{M}_{0}=\{0,1,2, \ldots, s\}$, where $\eta\left(i_{k}\right)=0$ indicates that there is no packet dropout during the time interval $\left(i_{k}, i_{k+1}\right]$ and $s$ represents the maximal length of consecutive packet losses. Denote $\mathscr{M}=$ $\mathscr{M}_{0} \backslash\{0\}$ the packet-loss subset of $\mathscr{M}_{0} .\left\{\eta\left(i_{k}\right), k \geqslant 0\right\}$ is assumed to be independent of $\left\{w_{t}\right\},\left\{v_{t}\right\}$ and $x_{0}$. The corresponding transition probability is

$$
\pi_{i j}:=\operatorname{Pr}\left(\eta\left(i_{k+1}\right)=j \mid \eta\left(i_{k}\right)=i\right) \geqslant 0
$$

and the initial distribution of $\eta(0)$ is: $\left\{\pi_{j}:=\operatorname{Pr}(\eta(0)=j), j \in \mathscr{M}_{0}\right\}$. Note that $\Pi:=\left(\pi_{i j}\right)_{(s+1) \times(s+1)}$ is a known matrix and for all $i \in \mathscr{M}_{0}, \sum_{j=0}^{s} \pi_{i j}=1$.

The state prediction error covariance matrix $P_{t}, t \geqslant 0$ can be written as

$$
P_{t}:=E\left[\left(x_{t}-\hat{x}_{t \mid t-1}\right)\left(x_{t}-\hat{x}_{t \mid t-1}\right)^{\prime}\right]
$$

where $\hat{x}_{t \mid t-1}$ stands for the optimal estimate at time $t$. Intuitively speaking, at each time step, the time-varying Kalman filter performs both the time and measurement updates when a packet arrives; otherwise it performs the time update only; see [12] for more details. As in [12], we use $\gamma_{t} \in\{0,1\}$ to represent the loss (with $\gamma_{t}=0$ ) or arrival (with $\gamma_{t}=1$ ) of single packet at each time point. Henceforth, we can derive the following random Riccati equation easily:

$$
P_{t+1}=A P_{t} A^{\prime}+B Q B^{\prime}-\gamma_{t} A P_{t} C^{\prime}\left(C P_{t} C^{\prime}+R\right)^{-1} C P_{t} A^{\prime}
$$

with initial condition

$$
P_{0}=E\left[\left(x_{0}-\hat{x}_{0 \mid-1}\right)\left(x_{0}-\hat{x}_{0 \mid-1}\right)^{\prime}\right]=E\left[x_{0} x_{0}^{\prime}\right]=\Sigma_{0}
$$

It is worth mentioning that the random process $\left\{\gamma_{t}, t \geqslant 0\right\}$, which is driven by the packet-loss process $\left\{\eta\left(i_{k}\right), k \geqslant 0\right\}$, is neither the i.i.d. Bernoulli process as in [12] nor the binary Markovian process like [13], and the stochastic characteristic of $\left\{\gamma_{t}, t \geqslant 0\right\}$ is still unknown although it is totally determined for any given realization of $\left\{\eta\left(i_{k}\right), k \geqslant 0\right\}$.

The notion of sojourn times is usually defined as follows. Set $\beta_{0}=0$ and

$$
\begin{array}{cc}
\alpha_{1}:=\inf \left\{i_{k}+1: i_{k} \geqslant \beta_{0}, \eta\left(i_{k}\right) \geqslant 1\right\}, & \beta_{1}:=\inf \left\{i_{k+1}: i_{k+1}>\alpha_{1}, \eta\left(i_{k}\right) \geqslant 1\right\} \\
\vdots & \vdots \\
\alpha_{l}:=\inf \left\{i_{k}+1: i_{k} \geqslant \beta_{l-1}, \eta\left(i_{k}\right) \geqslant 1\right\}, & \beta_{l}:=\inf \left\{i_{k+1}: i_{k+1}>\alpha_{l}, \eta\left(i_{k}\right) \geqslant 1\right\}
\end{array}
$$

thus, $\alpha_{l}, \beta_{l} \geqslant 1$ are two stopping times of $\left\{\gamma_{t}, t \geqslant 0\right\}$. The sojourn times are the lengths of the $l$ th excursion to the state 1 or 0 , as defined by:

$$
\alpha_{l}^{\star}:=\alpha_{l}-\beta_{l-1}, \quad \beta_{l}^{\star}:=\beta_{l}-\alpha_{l}, \quad l \geqslant 1
$$

To illustrate the notions of $\gamma_{t}, i_{k}, \eta\left(i_{k}\right), \alpha_{l}, \beta_{l}, \alpha_{l}^{\star}, \beta_{l}^{\star}$, we assume that there is one sample path of $\gamma_{t}$ as shown in Figure 1. Thus, the corresponding sequence $\left\{i_{k}, k \geqslant 0\right\}$ is given by

$$
\left\{i_{0}, i_{1}, \ldots, i_{14}\right\}=\{0,1,4,5,6,10,11,12,13,14,16,17,18,19,20\}
$$




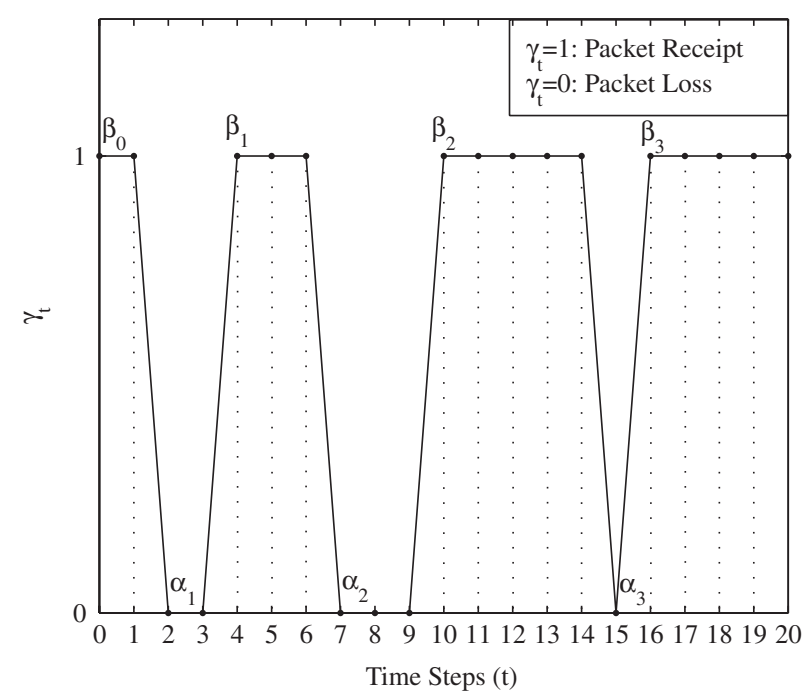

Figure 1. An example: one possible sample path of $\gamma_{t}$.

and by (4) we can get the packet-loss process as follows:

$$
\left\{\eta\left(i_{0}\right), \eta\left(i_{1}\right), \ldots, \eta\left(i_{13}\right)\right\}=\{0,2,0,0,3,0,0,0,0,1,0,0,0,0\}
$$

From the definition of stopping time and sojourn time, it is also easy to obtain:

$$
\begin{aligned}
& \left\{\alpha_{1}, \alpha_{2}, \alpha_{3}\right\}=\{2,7,15\}, \quad\left\{\beta_{0}, \beta_{1}, \beta_{2}, \beta_{3}\right\}=\{0,4,10,16\} \\
& \left\{\alpha_{1}^{\star}, \alpha_{2}^{\star}, \alpha_{3}^{\star}\right\}=\{2,3,5\}, \quad\left\{\beta_{1}^{\star}, \beta_{2}^{\star}, \beta_{3}^{\star}\right\}=\{2,3,1\}
\end{aligned}
$$

We recall the following definition of peak covariance stability introduced by Huang and Dey $[13,15]$.

\section{Definition 2.1}

The Kalman filter with packet losses or the random Riccati equation (5) is said to be peak covariance stable if $\sup _{l \geqslant 0} E\left[\left\|P_{\beta_{l}}\right\|\right]<+\infty$, where the expectation is taken with respect to the packet-loss process.

The sequence $\left\{P_{\beta_{l}}\right\}, l \geqslant 1$ is a collection of the prediction error covariance matrix after every consecutive packet losses (see also Figure 1). Note that for an unstable scalar model, $\left\{P_{\beta_{l}}\right\}$ gives the upper envelop of the covariance process [13]. For higher-order systems, intuitively, with the number of consecutive packet losses increases, one would expect that the filtering performance is getting worse although in general the covariance matrix may not necessarily be monotonically increasing over the period of packet losses. Hence, the peak covariance stability that captures the boundedness of $\sup _{l \geqslant 0} E\left[\left\|P_{\beta_{l}}\right\|\right]$ makes sense and is a reasonable measure of stability.

As any possible $P_{t}$ would be bounded if $\rho(A)<1[14]$, in the remainder of the paper we consider the non-trivial case: $\rho(A) \geqslant 1$. 


\section{MAIN RESULTS}

\subsection{Preliminaries}

First of all, if we define the general mapping $\Theta(\cdot)$ as

$$
\Theta(P, A, B, C, Q, R, S):=A P A^{\prime}+B Q B^{\prime}-\left(A P C^{\prime}+B S\right)\left(C P C^{\prime}+R\right)^{-1}\left(A P C^{\prime}+B S\right)^{\prime}
$$

then the Lyapunov recursion $P_{t+1}=G\left(P_{t}\right)$ and the Riccati recursion $P_{t+1}=F\left(P_{t}\right)$ with initial value $P_{0}$ can be generated by $\Theta$ as follows:

$$
\begin{aligned}
& G(P):=\lim _{\sigma^{2} \rightarrow \infty} \Theta\left(P, A, B, C, Q, \sigma^{2} I, 0\right)=A P A^{\prime}+B Q B^{\prime} \\
& F(P):=\Theta(P, A, B, C, Q, R, 0)=A P A^{\prime}+B Q B^{\prime}-A P C^{\prime}\left(C P C^{\prime}+R\right)^{-1} C P A^{\prime}
\end{aligned}
$$

where $P \in \mathscr{S}^{n}$.

The following lemma can be found in $[3,6,13]$.

\section{Lemma 1}

For $G(\cdot)$ and $F(\cdot)$, the statements below hold:

(i) both $G(\cdot)$ and $F(\cdot)$ are monotonically increasing, i.e. for any $P_{1} \geqslant P_{2} \geqslant 0$, we have $G\left(P_{1}\right) \geqslant G\left(P_{2}\right) \geqslant B Q B^{\prime}$ and $F\left(P_{1}\right) \geqslant F\left(P_{2}\right) \geqslant B Q B^{\prime}$

(ii) there exists a constant $K>0$ such that for any $P \in \mathscr{S}_{0}^{n}, F^{k}(P) \leqslant K I_{n}$ for all $k \geqslant I_{0}$, where $\mathscr{S}_{0}^{n}$ is a convex subset of $\mathscr{S}^{n}$ as

$$
\mathscr{S}_{0}^{n}:=\left\{P: 0 \leqslant P \leqslant G(\bar{P}) \text {, for some } \bar{P} \in \mathscr{S}^{n}\right\}
$$

where $F^{i}(P)$ denotes the result of the $i$ th recursion of (8);

(iii) there exists a constant $K>0$ such that for any $P \in \mathscr{S}^{n}, F^{k+1}(P) \leqslant K I_{n}$ for all $k \geqslant I_{\mathrm{o}}$;

(iv) if $I_{\mathrm{o}}=1$, i.e. $C$ is invertible, then $F(P) \leqslant A C^{-1} R\left(C^{-1}\right)^{\prime} A^{\prime}$, i.e. there exists a positive constant $d_{1}^{(0)}$ such that

$$
\|F(P)\| \leqslant d_{1}^{(0)}
$$

(v) if $I_{\mathrm{o}} \geqslant 2$, then for $1 \leqslant i \leqslant\left(I_{\mathrm{o}}-1\right)$, there exist positive constants $d_{i}^{(0)}$ and $d_{i}^{(1)}$ such that

$$
\left\|F^{i}(P)\right\| \leqslant d_{i}^{(1)}\|P\|+d_{i}^{(0)}
$$

It is indispensable to derive a systematic method for estimating $d_{i}^{(1)}$, due to its importance in checking the peak covariance stability. In the first place, we need the following lemma related to the so-called global approach; see [1, p. 539].

\section{Lemma 2}

The covariance of the estimate error at time step $t, t \geqslant 1$ from the standard Riccati recursion (8), i.e. $P_{t}=F^{t}\left(P_{0}\right)$, can be alternatively evaluated by

$$
P_{t}=\Theta\left(P_{0}, A^{(t)}, B^{(t)}, C^{(t)}, Q^{(t)}, D^{(t)} Q^{(t)}\left(D^{(t)}\right)^{\prime}+R^{(t)}, Q^{(t)}\left(D^{(t)}\right)^{\prime}\right)
$$


in one step, where $A^{(t)}, B^{(t)}, C^{(t)}, D^{(t)}, Q^{(t)}, R^{(t)}$ are defined as

$$
\begin{aligned}
& A^{(t)}=A^{t}, \quad B^{(t)}=\left[A^{t-1} B, A^{t-2} B, \ldots, B\right], \\
& D^{(1)}=0 \quad \text { for } t=1 ; \quad D^{(t)}=\left[\begin{array}{cccc}
0 & 0 & \cdots & 0 \\
C B & 0 & \cdots & 0 \\
\vdots & \vdots & \ddots & \vdots \\
C A^{t-2} B & C A^{t-3} B & \cdots & 0
\end{array}\right] \text { for } t \geqslant 2 \\
& Q^{(t)}=\operatorname{diag}(Q, Q, \ldots, Q), \quad R^{(t)}=\operatorname{diag}(R, R, \ldots, R)
\end{aligned}
$$

\section{Proof}

Suppose there is no packet dropout, thus we can put the state-space model (1), (2) into global form:

$$
\begin{aligned}
x_{t} & =A^{(t)} x_{0}+B^{(t)} \mathbf{w}^{(t)} \\
\mathbf{y}^{(t)} & =C^{(t)} x_{0}+D^{(t)} \mathbf{w}^{(t)}+\mathbf{v}^{(t)}=C^{(t)} x_{0}+\left[\begin{array}{ll}
D^{(t)} & I
\end{array}\right]\left[\begin{array}{c}
\mathbf{w}^{(t)} \\
\mathbf{v}^{(t)}
\end{array}\right]
\end{aligned}
$$

with $\mathbf{w}^{(t)}:=\operatorname{col}\left\{w_{0}, w_{1}, \ldots, w_{t-1}\right\}, \mathbf{y}^{(t)}:=\operatorname{col}\left\{y_{0}, y_{1}, \ldots, y_{t-1}\right\}, \mathbf{v}^{(t)}:=\operatorname{col}\left\{v_{0}, v_{1}, \ldots, v_{t-1}\right\}$, and

$$
\left\langle\left[\begin{array}{c}
\mathbf{w}^{(t)} \\
\mathbf{v}^{(t)} \\
x_{0}
\end{array}\right],\left[\begin{array}{c}
\mathbf{w}^{(t)} \\
\mathbf{v}^{(t)} \\
x_{0}
\end{array}\right]\right\rangle=\left[\begin{array}{ccc}
Q^{(t)} & 0 & 0 \\
0 & R^{(t)} & 0 \\
0 & 0 & \Sigma_{0}
\end{array}\right]
$$

The result of this lemma follows by the optimality of the Kalman filter. The only non-trivial thing here is the correlation between the process noise term and measurement noise term in the global model (11), (12), which can be solved by introducing some non-zero $S$ in the Riccati recursion.

It is easy to see $A^{(t)}, B^{(t)}, C^{(t)}, D^{(t)}$ are the state transition matrix, observability map, controllability map and the impulse response matrix, respectively. Based on the above lemma, it is possible to study some properties of $\left\{P_{t}, t \geqslant 0\right\}$ without resorting to the Riccati recursion (8) step by step explicitly.

\section{Lemma 3}

For $1 \leqslant i \leqslant\left(I_{\mathrm{o}}-1\right), I_{\mathrm{o}} \geqslant 2$, the positive constants $d_{i}^{(1)}$ in inequality (10) can be bounded by

$$
d_{i}^{(1)} \leqslant \underline{\lambda}_{i}
$$


and $\underline{\lambda}_{i}, 1 \leqslant i \leqslant\left(I_{\mathrm{o}}-1\right)$ are the solutions to the following set of convex optimization problems in terms of LMI:

$$
\begin{array}{lll}
\operatorname{minimize} & \lambda_{i} \\
\text { subject to } & {\left[\begin{array}{cc}
\lambda_{i} I & \left(A^{(i)}+K^{(i)} C^{(i)}\right)^{\prime} \\
A^{(i)}+K^{(i)} C^{(i)} & I
\end{array}\right] \geqslant 0}
\end{array}
$$

where $A^{(i)}, C^{(i)}$ are defined similar to Lemma 2 and $K^{(i)}$ are unknown parameter matrices with compatible dimensions.

Proof

First consider the case $i=1$. Similar to [12], let

$$
\psi(K, P):=(A+K C) P(A+K C)^{\prime}+K R K^{\prime}+B Q B^{\prime}
$$

By taking $\partial \psi(K, P) / \partial K$, it is easy to get that

$$
K=K_{P}:=-A P C^{\prime}\left(C P C^{\prime}+R\right)^{-1}
$$

minimizes $\psi(K, P)$ and $\psi\left(K_{P}, P\right)=F(P)$, which implies that

$$
\|F(P)\|=\left\|\psi\left(K_{P}, P\right)\right\| \leqslant\|\psi(K, P)\| \leqslant\|A+K C\|^{2}\|P\|+\left\|K R K^{\prime}+B Q B^{\prime}\right\|
$$

Compare the above inequality with (10), then we can conclude that $d_{1}^{(1)}$ can be bounded by $\|A+K C\|^{2}$ for any $K$, which further implies $d_{1}^{(1)} \leqslant \min _{K}\left\{\|A+K C\|^{2}\right\}$. We also have

$$
\|A+K C\|^{2}=\min \left\{\lambda_{1} \mid \lambda_{1} I-(A+K C)^{\prime}(A+K C) \geqslant 0\right\}
$$

and $\underline{\lambda}_{1}=\min _{K}\left\{\|A+K C\|^{2}\right\}$ can be easily obtained through (13) with $i=1$.

For $i>1$, we have $F^{i}(P)=\Theta\left(P, A^{(i)}, B^{(i)}, C^{(i)}, Q^{(i)}, D^{(i)} Q^{(i)}\left(D^{(i)}\right)^{\prime}+R^{(i)}, Q^{(i)}\left(D^{(i)}\right)^{\prime}\right)$ based on Lemma 2. Further denote $\bar{R}^{(i)}=D^{(i)} Q^{(i)}\left(D^{(i)}\right)^{\prime}+R^{(i)}, S^{(i)}=Q^{(i)}\left(D^{(i)}\right)^{\prime}$ and define the quantity

$$
\bar{\psi}\left(K^{(i)}, P\right):=\left(A^{(i)}+K^{(i)} C^{(i)}\right) P\left(A^{(i)}+K^{(i)} C^{(i)}\right)^{\prime}+\left[B^{(i)} K^{(i)}\right]\left[\begin{array}{cc}
Q^{(i)} & S^{(i)} \\
\left(S^{(i)}\right)^{\prime} & \bar{R}^{(i)}
\end{array}\right]\left[\begin{array}{c}
\left(B^{(i)}\right)^{\prime} \\
\left(K^{(i)}\right)^{\prime}
\end{array}\right]
$$

Therefore, $K^{(i)}=K_{P}^{(i)}:=-\left(A^{(i)} P\left(C^{(i)}\right)^{\prime}+B^{(i)} S^{(i)}\right)\left(C^{(i)} P\left(C^{(i)}\right)^{\prime}+\bar{R}^{(i)}\right)^{-1}$ minimizes $\bar{\psi}\left(K^{(i)}, P\right)$ and $\bar{\psi}\left(K_{P}^{(i)}, P\right)=F^{i}(P)$. We have

$$
\begin{aligned}
\left\|F^{i}(P)\right\| & =\left\|\bar{\psi}\left(K_{P}^{(i)}, P\right)\right\| \leqslant\left\|\bar{\psi}\left(K^{(i)}, P\right)\right\| \\
& \leqslant\left\|A^{(i)}+K^{(i)} C^{(i)}\right\|^{2}\|P\|+\left\|\left[\begin{array}{ll}
B^{(i)} & K^{(i)}
\end{array}\right]\left[\begin{array}{cc}
Q^{(i)} & S^{(i)} \\
\left(S^{(i)}\right)^{\prime} & \bar{R}^{(i)}
\end{array}\right]\left[\begin{array}{c}
\left(B^{(i)}\right)^{\prime} \\
\left(K^{(i)}\right)^{\prime}
\end{array}\right]\right\|
\end{aligned}
$$

Similarly, after comparing it with (10), we have $d_{i}^{(1)} \leqslant \underline{\lambda}_{i}=\min _{K^{(i)}}\left\{\left\|A^{(i)}+K^{(i)} C^{(i)}\right\|^{2}\right\}$ and (13) again offers a way to compute $\underline{\lambda}_{i}$ when $1<i \leqslant\left(I_{0}-1\right)$. This completes the proof of this lemma. 
Obviously, $\underline{\lambda}_{i}$ as upper bounds for $d_{i}^{(1)}$ can be obtained through (13) via standard LMI toolbox directly.

\section{Remark 3.1}

From the proof of Lemma 3, there always exists $K^{(i)}$ such that $d_{i}^{(1)}=0$ for $i \geqslant I_{0}$, since $(A, C)$ is assumed to be observable and thus $C^{(i)}$ has full-column rank when $i \geqslant I_{\mathrm{o}}$.

\section{Remark 3.2}

In the simulation example of [13], $\|F(P)\| \leqslant\left\|A A^{\prime}\right\|\|P\|$ was used to estimate $d_{1}^{(1)}$ in inequality (10); in [15], the same authors applied a suboptimal estimator to aid stability check and adopted a numerical method to search for a better estimation of $d_{1}^{(1)}$ for a two-dimensional example. The method in [14] does not involve estimating these constants and was shown to be less conservative in some cases; however, it approximates $F^{i}(P)$ by $G^{i}(P)$ when $i \in\left[1,\left(I_{0}-1\right)\right]$ with $I_{0} \geqslant 2$, which is still conservative. Compared with the aforementioned papers, Lemma 3 presents an easy and efficient way to estimate $d_{i}^{(1)}$; see also the first simulation example of this paper.

When the packet losses are driven by a binary Markovian process, the sojourn times are proved to be i.i.d.; see Lemma 2 in [13]. In this note, the stochastic characteristic of sojourn times with bounded Markovian packet losses is given in the following lemma.

\section{Lemma 4}

Denote the joint distribution of sojourn times $\alpha_{l}^{\star}, \beta_{l}^{\star}$ by

$$
\operatorname{Pr}(l):=\operatorname{Pr}\left(\alpha_{1}^{\star}=a_{1}, \beta_{1}^{\star}=b_{1}, \alpha_{2}^{\star}=a_{2}, \beta_{2}^{\star}=b_{2}, \ldots, \alpha_{l}^{\star}=a_{l}, \beta_{l}^{\star}=b_{l}\right)
$$

where for any positive integer $i, a_{i} \in \mathscr{N}$ and $b_{i} \in \mathscr{M}$. Then, we have:

$$
\begin{aligned}
\operatorname{Pr}(1) & = \begin{cases}\pi_{b_{1}} & \text { if } a_{1}=1 \\
\pi_{0}\left(\pi_{00}\right)^{a_{1}-2} \pi_{0 b_{1}} & \text { if } a_{1} \geqslant 2\end{cases} \\
\operatorname{Pr}(l+1) & = \begin{cases}\pi_{b_{l} b_{l+1}} \operatorname{Pr}(l) & \text { if } a_{l+1}=1 \\
\pi_{b_{l} 0}\left(\pi_{00}\right)^{a_{l+1}-2} \pi_{0 b_{l+1}} \operatorname{Pr}(l) & \text { if } a_{l+1} \geqslant 2\end{cases}
\end{aligned}
$$

Proof

The result can be obtained based on the Markov property of the packet-loss process $\left\{\eta\left(i_{k}\right)\right\}$.

\subsection{Peak covariance stability}

Now we are in the position to present our main results. We start with considering the peak covariance stability for the special case: $I_{\mathrm{O}}=1$.

\section{Theorem 3.1}

If the observability index $I_{\mathrm{O}}$ of the observable linear system or the pair $(A, C)$ equals to 1 , then the Kalman filtering with bounded Markovian packet losses is always peak covariance stable. 
Proof

First of all, $E\left[\left\|P_{\beta_{0}}\right\|\right]=\left\|P_{0}\right\|$ since $P_{0} \in \mathscr{S}^{n}$ is a given constant matrix. Let $\left\{a_{l}, b_{l}, l \geqslant 1, a_{l} \in \mathscr{N}, b_{l} \in\right.$ $\mathscr{M}\}$ denote one realization of the random process $\left\{\alpha_{l}^{\star}, \beta_{l}^{\star}, l \geqslant 1\right\}$. As $P_{\beta_{1}}$ is uniquely determined for fixed $\alpha_{1}^{\star}, \beta_{1}^{\star}$, and $P_{0}$, we have:

$$
\begin{aligned}
E\left[\left\|P_{\beta_{1}}\right\|\right] & =\sum_{a_{1}=1}^{+\infty} \sum_{b_{1}=1}^{s}\left\|P_{\beta_{1}}\right\| \operatorname{Pr}(1) \\
& =\sum_{a_{1}=2}^{+\infty} \sum_{b_{1}=1}^{s} \pi_{0}\left(\pi_{00}\right)^{a_{1}-2} \pi_{0 b_{1}}\left\|G^{b_{1}}\left(F^{a_{1}}\left(P_{0}\right)\right)\right\|+\sum_{b_{1}=1}^{s} \pi_{b_{1}}\left\|G^{b_{1}}\left(F\left(P_{0}\right)\right)\right\|
\end{aligned}
$$

In the above, Lemma 4 has been applied to obtain (14), whose first part describes all the possible trajectories starting from initial condition $\eta_{0}=0$ and reaching $\beta_{1}^{\star}=b_{1}, b_{1} \in \mathscr{M}$ before the first peak time $\beta_{1}$, and the second part corresponds to $\eta_{0}=b_{1}, b_{1} \in \mathscr{M}$. Then, by applying Lemma 1 , we have

$$
\begin{aligned}
E\left[\left\|P_{\beta_{1}}\right\|\right] \leqslant & \sum_{b_{1}=1}^{s} \sum_{a_{1}=2}^{+\infty} \pi_{0}\left(\pi_{00}\right)^{a_{1}-2} \pi_{0 b_{1}}\left\|G^{b_{1}}\left(K I_{n}\right)\right\| \\
& +\sum_{b_{1}=1}^{s} \pi_{b_{1}}\left(d_{1}^{(1)}\left\|A^{b_{1}}\right\|^{2}\left\|P_{0}\right\|+d_{1}^{(0)}\left\|A^{b_{1}}\right\|^{2}+c_{1}\right) \\
\leqslant & \sum_{b_{1}=1}^{s} \sum_{a_{1}=2}^{+\infty} c_{2} \pi_{0} \pi_{0 b_{1}}\left(\pi_{00}\right)^{a_{1}-2} \\
& +\sum_{b_{1}=1}^{s} \pi_{b_{1}}\left(d_{1}^{(1)}\left\|A^{b_{1}}\right\|^{2}\left\|P_{0}\right\|+d_{1}^{(0)}\left\|A^{b_{1}}\right\|^{2}+c_{1}\right) \\
\leqslant & c_{3}
\end{aligned}
$$

where $c_{1}, c_{2}$ are constants and $c_{3}$ is a function of $P_{0}$. Note that if $\pi_{00}=1$, i.e. it is the trivial case, the first part of (17) is 0 due to $\pi_{0 b_{1}}=0$ and it is convergent for $\pi_{00}<1$.

Furthermore, for any $l \geqslant 1$ we can deduce that

$$
\begin{aligned}
E\left[\left\|P_{\beta_{l+1}}\right\|\right]= & \sum_{a_{1}=1}^{+\infty} \sum_{b_{1}=1}^{s} \ldots \sum_{a_{l+1}=1}^{+\infty} \sum_{b_{l+1}=1}^{s}\left\|P_{\beta_{l+1}}\right\| \operatorname{Pr}(l+1) \\
= & \sum_{a_{1}=1}^{+\infty} \sum_{b_{1}=1}^{s} \ldots \sum_{a_{l+1}=2}^{+\infty} \sum_{b_{l+1}=1}^{s} \pi_{b_{l} 0}\left(\pi_{00}\right)^{a_{l+1}-2} \pi_{0 b_{l+1}}\left\|G^{b_{l+1}}\left(F^{a_{l+1}}\left(P_{\beta_{l}}\right)\right)\right\| \operatorname{Pr}(l) \\
& +\sum_{a_{1}=1}^{+\infty} \sum_{b_{1}=1}^{s} \ldots \sum_{b_{l+1}=1}^{s} \pi_{b_{l} b_{l+1}}\left\|G^{b_{l+1}}\left(F\left(P_{\beta_{l}}\right)\right)\right\| \operatorname{Pr}(l)
\end{aligned}
$$


Then, by using Lemma 1 again, the following inequalities can be obtained:

$$
\begin{aligned}
E\left[\left\|P_{\beta_{l+1}}\right\|\right] \leqslant & \sum_{a_{1}=1}^{+\infty} \sum_{b_{1}=1}^{s} \ldots \sum_{a_{l+1}=2}^{+\infty} \sum_{b_{l+1}=1}^{s} \pi_{b_{l} 0}\left(\pi_{00}\right)^{a_{l+1}-2} \pi_{0 b_{l+1}}\left\|G^{b_{l+1}}\left(K I_{n}\right)\right\| \operatorname{Pr}(l) \\
& +\sum_{a_{1}=1}^{+\infty} \sum_{b_{1}=1}^{s} \ldots \sum_{b_{l+1}=1}^{s} \pi_{b_{l} b_{l+1}}\left\|G^{b_{l+1}}\left(K I_{n}\right)\right\| \operatorname{Pr}(l) \\
\leqslant & \sum_{a_{1}=1}^{+\infty} \sum_{b_{1}=1}^{s} \ldots \sum_{a_{l+1}=2}^{+\infty} \sum_{b_{l+1}=1}^{s} c_{4} \pi_{b_{l} 0} \pi_{0 b_{l+1}}\left(\pi_{00}\right)^{a_{l+1}-2} \operatorname{Pr}(l) \\
& +\sum_{a_{1}=1}^{+\infty} \sum_{b_{1}=1}^{s} \ldots \sum_{b_{l+1}=1}^{s} c_{5} \pi_{b_{l} b_{l+1}} \operatorname{Pr}(l) \\
\leqslant & \sum_{a_{1}=1}^{+\infty} \sum_{b_{1}=1}^{s} \ldots \sum_{a_{l}=1}^{+\infty} \sum_{b_{l}=1}^{s} c_{6} \operatorname{Pr}(l)+\sum_{a_{1}=1}^{+\infty} \sum_{b_{1}=1}^{s} \ldots \sum_{a_{l}=1}^{+\infty} \sum_{b_{l}=1}^{s} c_{7} \operatorname{Pr}(l) \\
\leqslant & c_{8}
\end{aligned}
$$

where $c_{4}, \ldots, c_{8}$ are constants, and (18) is due to the fact that for $l \geqslant 1$, any possible value of $P_{\beta_{1}}$ belongs to $\mathscr{S}_{0}^{n}$. Hence, the two inequalities: $E\left[\left\|P_{\beta_{1}}\right\|\right] \leqslant c_{3}$ and $E\left[\left\|P_{\beta_{l+1}}\right\|\right] \leqslant c_{8}$ complete the proof of this theorem.

\section{Remark 3.3}

By the above theorem, we can conclude that every Kalman filter with bounded Markovian packet losses is peak covariance stable for observable scalar systems, since their observability index always equals to 1 .

The following theorem will extend the peak covariance stability result to $I_{0} \geqslant 2$.

\section{Theorem 3.2}

When the observability index $I_{0} \geqslant 2$, then the Kalman filtering with bounded Markovian packet losses is peak covariance stable, if $\rho(H)<1$, where $H=\left(h_{i j}\right)_{s \times s}$ and

$$
h_{i j}=\left(\pi_{i j} d_{1}^{(1)}+\sum_{m=2}^{I_{\mathrm{o}}-1} \pi_{i 0}\left(\pi_{00}\right)^{m-2} \pi_{0 j} d_{m}^{(1)}\right)\left\|A^{j}\right\|^{2}
$$

Proof

By a similar argument as in Theorem 1, we have:

$$
\begin{aligned}
E\left[\left\|P_{\beta_{1}}\right\|\right]= & \sum_{a_{1}=1}^{+\infty} \sum_{b_{1}=1}^{s}\left\|P_{\beta_{1}}\right\| \operatorname{Pr}(1) \\
= & \sum_{b_{1}=1}^{s} \sum_{a_{1}=I_{0}+1}^{+\infty} \pi_{0}\left(\pi_{00}\right)^{a_{1}-2} \pi_{0 b_{1}}\left\|G^{b_{1}}\left(F^{a_{1}}\left(P_{0}\right)\right)\right\| \\
& +\sum_{b_{1}=1}^{s} \sum_{a_{1}=2}^{I_{0}} \pi_{0}\left(\pi_{00}\right)^{a_{1}-2} \pi_{0 b_{1}}\left\|G^{b_{1}}\left(F^{a_{1}}\left(P_{0}\right)\right)\right\|+\sum_{b_{1}=1}^{s} \pi_{b_{1}}\left\|G^{b_{1}}\left(F\left(P_{0}\right)\right)\right\|
\end{aligned}
$$


Then from Lemma 1, we get

$$
\begin{aligned}
E\left[\left\|P_{\beta_{1}}\right\|\right] \leqslant & \sum_{b_{1}=1}^{s} \pi_{b_{1}}\left(d_{1}^{(1)}\left\|A^{b_{1}}\right\|^{2}\left\|P_{0}\right\|+d_{1}^{(0)}\left\|A^{b_{1}}\right\|^{2}+e_{2}\right) \\
& +\sum_{b_{1}=1}^{s} \sum_{a_{1}=I_{0}+1}^{+\infty} \pi_{0}\left(\pi_{00}\right)^{a_{1}-2} \pi_{0 b_{1}}\left\|G^{b_{1}}\left(K I_{n}\right)\right\| \\
& +\sum_{b_{1}=1}^{s} \sum_{a_{1}=2}^{I_{0}} \pi_{0}\left(\pi_{00}\right)^{a_{1}-2} \pi_{0 b_{1}}\left(d_{a_{1}}^{(1)}\left\|A^{b_{1}}\right\|^{2}\left\|P_{0}\right\|+d_{a_{1}}^{(0)}\left\|A^{b_{1}}\right\|^{2}+e_{1}\right) \\
\leqslant & e_{3}+\sum_{b_{1}=1}^{s}\left(\sum_{a_{1}=2}^{I_{0}} \pi_{0}\left(\pi_{00}\right)^{a_{1}-2} \pi_{0 b_{1}} d_{a_{1}}^{(1)}+\pi_{b_{1}} d_{1}^{(1)}\right)\left\|A^{b_{1}}\right\|^{2}\left\|P_{0}\right\| \\
= & e_{3}+F D
\end{aligned}
$$

where $e_{1}, e_{2}, e_{3}$ are constants, $D=\left[\begin{array}{llll}1 & 1 & \ldots & 1\end{array}\right]^{\mathrm{T}} \in \mathscr{R}^{s \times 1}, F=\left[\begin{array}{llll}\phi_{1} & \phi_{2} & \ldots & \phi_{s}\end{array}\right] \in \mathscr{R}^{1 \times s}$ and for $i=$ $1,2, \ldots, s$

$$
\phi_{i}=\left(\pi_{i} d_{1}^{(1)}+\sum_{m=2}^{I_{\mathrm{o}}} \pi_{0}\left(\pi_{00}\right)^{m-2} \pi_{0 i} d_{m}^{(1)}\right)\left\|A^{i}\right\|^{2}\left\|P_{0}\right\|
$$

For every $l \geqslant 1$, we have:

$$
\begin{aligned}
E\left[\left\|P_{\beta_{l+1}}\right\|\right]= & \sum_{a_{1}=1}^{+\infty} \sum_{b_{1}=1}^{s} \ldots \sum_{a_{l+1}=1}^{+\infty} \sum_{b_{l+1}=1}^{s}\left\|P_{\beta_{l}+1}\right\| \operatorname{Pr}(l+1) \\
= & \sum_{a_{1}=1}^{+\infty} \sum_{b_{1}=1}^{s} \ldots \sum_{b_{l+1}=1}^{s} \sum_{a_{l+1}=I_{0}}^{+\infty} \pi_{b_{l} 0}\left(\pi_{00}\right)^{a_{l+1}-2} \pi_{0 b_{l+1}}\left\|G^{b_{l+1}}\left(F^{a_{l+1}}\left(P_{\beta_{l}}\right)\right)\right\| \operatorname{Pr}(l) \\
& +\sum_{a_{1}=1}^{+\infty} \sum_{b_{1}=1}^{s} \ldots \sum_{b_{l+1}=1}^{s} \sum_{a_{l+1}=2}^{I_{0}-1} \pi_{b_{l} 0}\left(\pi_{00}\right)^{a_{l+1}-2} \pi_{0 b_{l+1}}\left\|G^{b_{l+1}}\left(F^{a_{l+1}}\left(P_{\beta_{l}}\right)\right)\right\| \operatorname{Pr}(l) \\
& +\sum_{a_{1}=1}^{+\infty} \sum_{b_{1}=1}^{s} \ldots \sum_{b_{l+1}=1}^{s} \pi_{b_{l} b_{l+1}}\left\|G^{b_{l+1}}\left(F\left(P_{\beta_{l}}\right)\right)\right\| \operatorname{Pr}(l) \\
:= & \Gamma_{1}+\Gamma_{2}+\Gamma_{3}
\end{aligned}
$$

It is easy to estimate that

$$
\begin{aligned}
\Gamma_{1} \leqslant & \sum_{a_{1}=1}^{+\infty} \sum_{b_{1}=1}^{s} \ldots \sum_{b_{l+1}=1}^{s} \sum_{a_{l+1}=I_{0}}^{+\infty} \pi_{b_{l} 0}\left(\pi_{00}\right)^{a_{l+1}-2} \pi_{0 b_{l+1}}\left\|G^{b_{l+1}}\left(K I_{n}\right)\right\| \operatorname{Pr}(l) \leqslant e_{4} \\
\Gamma_{2} \leqslant & \sum_{a_{1}=1}^{+\infty} \sum_{b_{1}=1}^{s} \ldots \sum_{b_{l+1}=1}^{s} \sum_{a_{l+1}=2}^{I_{0}-1} \pi_{b_{l} 0}\left(\pi_{00}\right)^{a_{l+1}-2} \pi_{0 b_{l+1}} \\
& \times\left(d_{a_{l+1}}^{(1)}\left\|A^{b_{l+1}}\right\|^{2}\left\|P_{\beta_{l}}\right\|+d_{a_{l+1}}^{(0)}\left\|A^{b_{l+1}}\right\|^{2}+e_{5}\right) \operatorname{Pr}(l) \\
\Gamma_{3} \leqslant & \sum_{a_{1}=1}^{+\infty} \sum_{b_{1}=1}^{s} \ldots \sum_{b_{l+1}=1}^{s} \pi_{b_{l} b_{l+1}}\left(d_{1}^{(1)}\left\|A^{b_{l+1}}\right\|^{2}\left\|P_{\beta_{l}}\right\|+d_{1}^{(0)}\left\|A^{b_{l+1}}\right\|^{2}+e_{6}\right) \operatorname{Pr}(l)
\end{aligned}
$$


with constants $e_{4}, e_{5}, e_{6}$ and it follows recursively that

$$
E\left[\left\|P_{\beta_{l+1}}\right\|\right] \leqslant F H^{l} D+e_{7}
$$

where $e_{7}$ is a constant and $H$ is described in Equation (18).

Based on (22), if $\rho(H)<1, E\left[\left\|P_{\beta_{l+1}}\right\|\right]$ is bounded as $l \rightarrow+\infty$, which implies that $\sup _{l \geqslant 1} E\left[\left\|P_{\beta_{l}}\right\|\right]<+\infty$. This completes the proof.

Note that when the observability index $I_{\mathrm{o}}=2$, the second item in the parenthesis of (18) vanishes.

Remark 3.4

In [4], the authors presented a modified observer-based estimator running parallel with the Kalman filter. The scheme can reduce the error covariance by switching to the suboptimal estimator when the error covariance of the Kalman filter is above some bound due to packet losses; however, this advantage is achieved at the expenses of sending a series of previous measurements through the network at each time step. Another difference is that the metric they considered is $\operatorname{Pr}\left[P_{t} \leqslant M\right]$ with upper-bound matrix $M$ instead of $E\left[P_{t}\right]$ or $E\left[\left\|P_{\beta_{l}}\right\|\right]$ adopted in this paper. The relationship between these two metrics could be an interesting topic for future studies.

Remark 3.5

From (18), it can be observed that if the maximum number of consecutive packet dropouts $s$ is large but transition probabilities $\pi_{0 j}$ and $\pi_{i j}$ are very small for large values of $i$ and $j$ and the system does not grow very fast, the stability condition of Theorem 2 may still be dominated by the part of the matrix $H$ corresponding to lower values of consecutive packet dropouts.

\section{NUMERICAL EXAMPLES}

\subsection{Case I: a second-order system}

Consider the same system model as in $[13,14]$ :

$$
A=\left[\begin{array}{cc}
1.3 & 0.3 \\
0 & 1.2
\end{array}\right], \quad C=\left[\begin{array}{ll}
1 & 1
\end{array}\right]
$$

The covariance of $w(t), v(t)$ are chosen as $Q=I_{2}, R=1$. First, we consider the packet-loss model of $[13,14]$ where the packet loss at each time step is a Markov chain, which implies that the number of consecutive packet losses could be infinite. In this situation, it is easy to check that $I_{\mathrm{o}}=2$ and we need to estimate $d_{1}^{(1)}$ only. From Lemma 3, we have $d_{1}^{(1)}=1.2200$, which is much smaller than the estimation: 2.00813 in [13]. Further, if we apply Lemma 3 of this note to Theorem 3.1 in [14], the condition there can be rewritten as

$$
\sum_{i=1}^{\infty}\left\|A^{i}\right\|^{2}(1-q)^{i-1} q \sum_{j=1}^{I_{0}-1} d_{j}^{(1)}(1-p)^{j-1} p<1
$$

then for the same recovery rate $q=0.65$, we can easily obtain that the maximum failure rate $p$ is 0.1960 , which also shows that the estimation in Lemma 3 is better than that of [14] where the maximum failure rate $p$ is 0.1191 . 

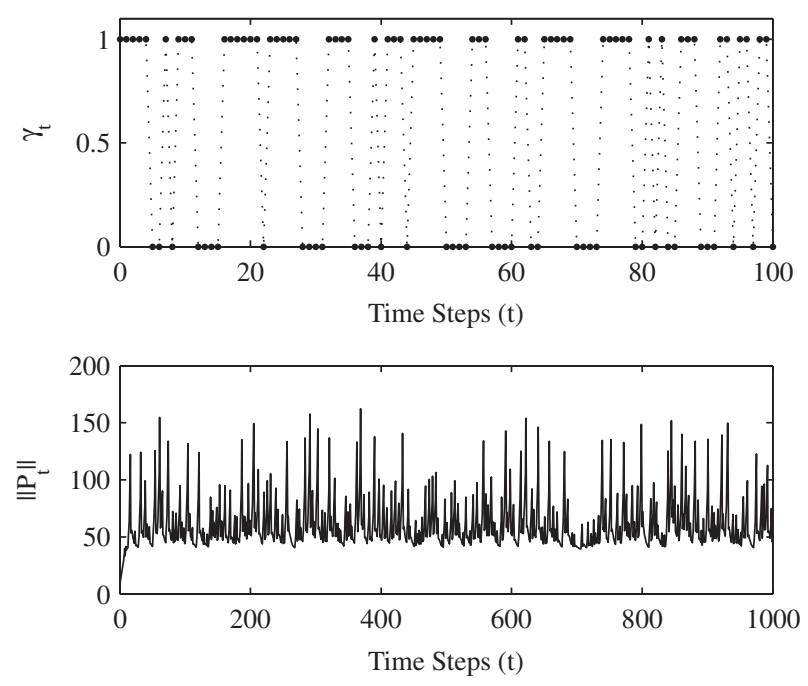

Figure 2. Case I: $\gamma_{t}$ and $\left\|P_{t}\right\|$ vs time steps.

Next, we consider the bounded Markovian packet-loss case. Suppose the maximum successive packet losses of the underlying network is 2 and the transition probability matrix is given by:

$$
\Pi_{1}=\left[\begin{array}{lll}
0.6 & 0.2 & 0.2 \\
0.8 & 0.1 & 0.1 \\
0.8 & 0.1 & 0.1
\end{array}\right]
$$

From Theorem 2, we have $\rho(H)=0.7352<1$; thus, the Kalman filtering is peak covariance stable. Figure 2 illustrates a sample path of $\gamma_{t}$ and $\left\|P_{t}\right\|$ with the given $\Pi_{1}$.

Moreover, if the maximum number of successive packet losses shrinks to 1 with transition probability matrix

$$
\Pi_{2}=\left[\begin{array}{ll}
0.6 & 0.4 \\
0.8 & 0.2
\end{array}\right]
$$

then $\rho(H)=0.4900<1$. Hence, we can conclude that the Kalman filtering is peak covariance stable as well.

\subsection{Case II: a third-order system}

Consider the following third-order system:

$$
A=\left[\begin{array}{ccc}
0 & 0.1 & 1.2 \\
0 & 0.5 & 0 \\
1.3 & 0.1 & 0
\end{array}\right], \quad C=\left[\begin{array}{lll}
1 & 0 & 0
\end{array}\right]
$$



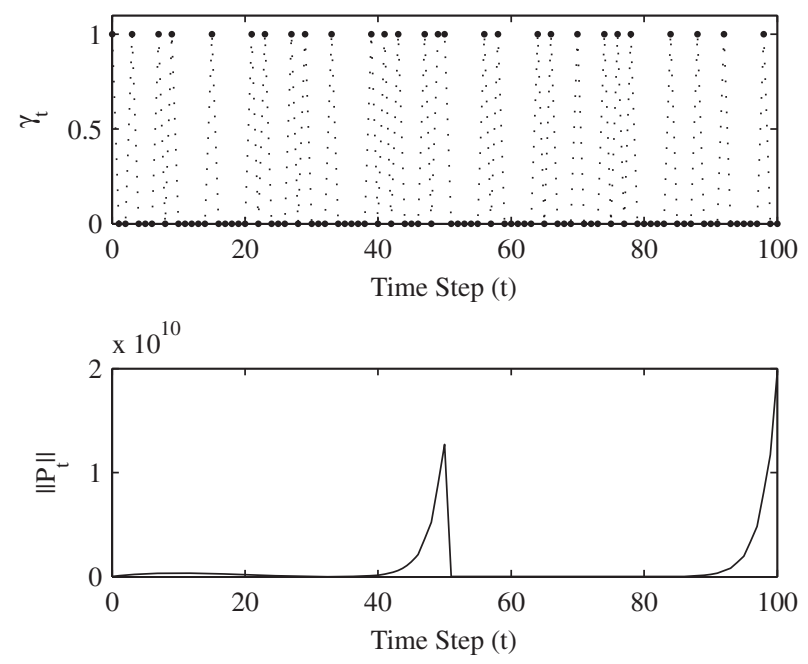

Figure 3. Case II $\left(C=\left[\begin{array}{lll}1 & 0 & 0\end{array}\right]\right): \gamma_{t}$ and $\left\|P_{t}\right\|$ vs time steps.

with $Q=I_{3}, R=1$. The eigenvalues of $A$ are $1.2490,-1.2490,0.5000$, and the observability index $I_{\mathrm{O}}$ is 3 . Suppose the maximum consecutive packet losses here is 5 and

$$
\Pi=\left[\begin{array}{cccccc}
0.5 & 0.1 & 0.1 & 0.1 & 0.1 & 0.1 \\
0.1 & 0.4 & 0 & 0.3 & 0 & 0.2 \\
0.1 & 0.3 & 0.1 & 0.3 & 0 & 0.2 \\
0.1 & 0.4 & 0 & 0.3 & 0 & 0.2 \\
0.1 & 0.3 & 0 & 0.3 & 0.1 & 0.2 \\
0.1 & 0.4 & 0 & 0.3 & 0 & 0.2
\end{array}\right]
$$

After obtaining $d_{1}^{(1)}=1.4522, d_{2}^{(1)}=0.0933$ from Lemma 3 , we have $\rho(H)=5.7932>1$ by Theorem 2 again, and Figure 3 shows that a sample path of $\left\|P_{t}\right\|$ can reach a relatively high level even before 100 time steps. Note that the rapid drop of $\left\|P_{t}\right\|$ around the 50th time step is mainly due to the double successive packet receipts.

Furthermore, if we set the matrix $C=I_{3}$ with $I_{0}=1$, then according to Theorem 1, no additional condition is needed for the peak covariance stability. Figure 4 gives a sample path of $\gamma_{t}$ and $\left\|P_{t}\right\|$ with the same $\Pi$.

\section{CONCLUSIONS}

The main contributions of this paper include: (1) a less conservative approach to the upper-bound estimation of Riccati-recursion solution; (2) some sufficient stability condition for time-varying Kalman filter with finite consecutive packet losses driven by a Markov chain. Two numerical examples were presented to illustrate the usefulness of the derived results. It is worth noting that 

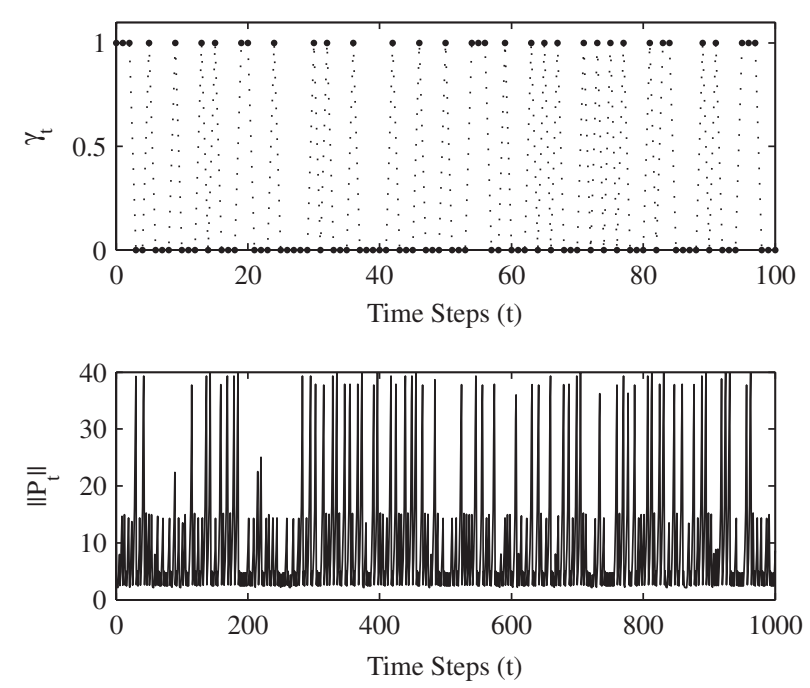

Figure 4. Case II $\left(C=I_{3}\right): \gamma_{t}$ and $\left\|P_{t}\right\|$ vs time steps.

the sufficient condition may still be conservative and better stability conditions could be a future research topic. Conditions for covariance stability in a usual sense and the relationship between the peak covariance stability and usual covariance stability deserve future studies. The corresponding control problem for networked systems with possible packet losses is another interesting topic worth of investigation.

\section{ACKNOWLEDGEMENTS}

The authors would like to thank the anonymous reviewers for their constructive comments and suggestions.

\section{REFERENCES}

1. Kailath T, Sayed A, Hassibi B. Linear Estimation. Prentice-Hall: Upper Saddle River, NJ, 2000.

2. Simon D. Optimal State Estimation: Kalman, $H_{\infty}$, and Nonlinear Approaches. Wiley-Interscience: New York, 2006.

3. Shi L, Epstein M, Tiwari A, Murray R. Estimation with information loss: asymptotic analysis and error bounds. 44th IEEE Conference on Decision and Control and European Control Conference, Seville, Spain, 2005; 1215-1221.

4. Epstein M, Shi L, Tiwari A, Murray R. Probabilistic performance of state estimation across a lossy network. Automatica 2008; in press. Available from: http//www.cds.caltech.edu/ epstein/Professional/Publications/ MEpstein-Estimation-Automatica2006.html.

5. Tiwari A, Jun M, Jeffcoat D, Murray R. Analysis of dynamic sensor coverage problem using Kalman filters for estimation. Proceedings of the 16th IFAC World Congress, Prague, Czech Republic, 2005.

6. Gupta V, Chung T, Hassibi B, Murray R. On a stochastic sensor selection algorithm with applications in sensor scheduling and sensor coverage. Automatica 2006; 42(2):251-260.

7. Zhang W, Branicky M, Phillips S. Stability of networked control systems. IEEE Control Systems Magazine 2001; 21(1):84-99.

8. Xie L, Soh Y. Robust Kalman filtering for uncertain systems. Systems and Control Letters 1994; 22(2):123-129.

9. Xie L, Soh Y, de Souza C. Robust Kalman filtering for uncertain discrete-time systems. IEEE Transactions on Automatic Control 1994; 39(6):1310-1314. 
10. Garcia G, Tarbouriech S, Peres P. Robust Kalman filtering for uncertain discrete-time linear systems. International Journal of Robust and Nonlinear Control 2003; 13(13):1225-1238.

11. Fletcher A, Rangan S, Goyal V. Estimation from lossy sensor data: jump linear modeling and Kalman filtering. Proceedings of the 3rd International Symposium on Information Processing in Sensor Networks, California, 2004; 251-258.

12. Sinopoli B, Schenato L, Franceschetti M, Poolla K, Jordan M, Sastry S. Kalman filtering with intermittent observations. IEEE Transactions on Automatic Control 2004; 49(9):1453-1464.

13. Huang M, Dey S. Stability of Kalman filtering with Markovian packet losses. Automatica 2007; 43(4):598-607.

14. Xie L, Xie L. Peak covariance stability of a random Riccati equation arising from Kalman filtering with observation losses. Journal of Systems Science and Complexity 2007; 20(2):262-272.

15. Huang M, Dey S. Kalman filtering with Markovian packet losses and stability criteria. 45th IEEE Conference on Decision and Control, San Diego, CA, 2006; 5621-5626.

16. Yu M, Wang L, Xie G, Chu T. Stabilization of networked control systems with data packet dropout via switched system approach. IEEE International Symposium on Computer Aided Control Systems Design, Taibei, China, 2004; 362-367.

17. Xiong J, Lam J. Stabilization of linear systems over networks with bounded packet loss. Automatica 2007; 43(1):80-87.

18. Chen Y, Xiao B, Xu Z. Fault detection filter design for networked control system with communication delays. Proceedings of the 6th International Conference on Intelligent Systems Design and Applications, Jinan, China, 2006; 867-872. 